\title{
p63, CK7, PAX8 and INI-1: an optimal immunohistochemical panel to distinguish poorly differentiated urothelial cell carcinoma from high-grade tumours of the renal collecting system
}

\author{
Jason C Carvalho, ${ }^{1}$ Dafydd G Thomas, ${ }^{1}$ Jonathan B McHugh, ${ }^{1}$ Rajal B Shah $^{2}$ \& \\ Lakshmi P Kunju ${ }^{1}$ \\ ${ }^{1}$ Department of Pathology, University of Michigan Medical Center, Ann Arbor, MI, USA, and ${ }^{2}$ Division of Urologic \\ Pathology, Caris Life Sciences, Irving, TX, USA
}

Date of submission 19 April 2011

Accepted for publication 16 August 2011

Carvalho J C, Thomas D G, McHugh J B, Shah R B \& Kunju L P

(2012) Histopathology 60, 597-608

\section{p63, CK7, PAX8 and INI-1: an optimal immunohistochemical panel to distinguish poorly differentiated urothelial cell carcinoma from high-grade tumours of the renal collecting system}

Aims: High-grade, poorly differentiated, infiltrative carcinomas involving the renal sinus region often pose challenging differential diagnostic considerations, specifically differentiation of urothelial carcinoma (UC) from renal cell carcinoma (RCC) subtypes. Accurate classification, especially the distinction of UC from RCC, is critical, as therapeutic approaches differ.

Methods and results: Cluster analysis was performed on immunohistochemical data from 18 invasive UCs, six CDCs, two RMCs, 18 type 2 papillary renal cell carcinomas (PRCCs) and 20 high-grade clear cell renal cell carcinomas (CRCCs) using a broad panel of traditional and novel immunohistochemical markers. The initial analysis with all antibodies segregates almost all the RCCs (45 of 46, 98\%) from all the UCs based on the lack of expression of p63 in all $(100 \%)$ RCCs, along with predominant strong expression of paired box gene 8 (PAX8) and vimentin, predominant lack of expression of high molecular weight cytoker- atin (HMCK) and CK7 and variable expression of RCC, CD10, CA1X and PAX2. All the UCs cluster together with strong, diffuse reactivity for p63, predominant reactivity for CK7 and high molecular weight kininogen (HMWK), and absent to minimal staining with PAX8, RCC antigen, PAX2, alpha-methylacyl-CoA racemase (AMACR), carbonic anhydrase IX (CAIX) and vimentin. After removing antibodies with significant overlap and/or minimal impact, a second analysis with a limited panel including p63, CK7, vimentin, integrase interactor 1 (INI-1) and PAX8 was performed. Again, the majority of UCs cluster into one group and p63 positivity separates all UCs from RCCs.

Conclusions: Lack of INI-1 expression, noted exclusively in RMCs, segregates RMCs into a separate cluster. PAX8 is rarely positive $(17 \%)$ in $\mathrm{UC}$, is commonly expressed in CDC, RMC, PRCC and CRCC and is superior to PAX2.

Keywords: collecting duct carcinoma, immunohistochemistry, p63, PAX8, renal medullary carcinoma, urothelial carcinoma

Abbreviations: AMACR, alpha-methylacyl-CoA racemase; CAIX, carbonic anhydrase IX; CDC, carcinoma of collecting ducts of Bellini; CRCC, clear cell renal cell carcinoma; HMCK, high molecular weight cytokeratin; INI-1, integrase interactor 1; PAX8, paired box gene 8; PRCC, papillary renal cell carcinoma; RCC, renal cell carcinoma; RMC, renal medullary carcinoma; S100A1, S100 calcium-binding protein A1; TMA, tissue microarray; UC, urothelial carcinoma 


\section{Introduction}

High-grade poorly differentiated carcinomas involving the renal sinus region, including the renal medulla and/or collecting duct system, are often diagnostically challenging. The distinction of invasive high-grade urothelial carcinoma (UC) involving the upper urinary tract from high-grade renal cell carcinomas (RCC), notably collecting duct renal cell carcinoma (carcinoma of collecting ducts of Bellini, CDC) and renal medullary carcinomas (RMC), can be especially difficult. While UC of the upper urinary tract, CDC and RMC are usually centred in the renal medullary/sinus region, other highgrade RCCs, including clear cell type renal cell carcinoma (CRCC) and papillary RCC (commonly type 2 PRCC), may extend to involve the renal sinus fat.

Although careful morphological examination will allow for the correct diagnosis in the majority of cases, there is sufficient overlap between these entities such that immunohistochemistry is often required to arrive at the correct diagnosis with confidence. Accurate characterization of these entities, specifically distinguishing UC from various subtypes of RCC, is critical, as therapeutic and prognostic implications differ.

Both CDC and RMC arise in the renal medulla and are located in the central region of the kidney. While CDCs are primarily high-grade adenocarcinomas with glandular architecture and occur predominantly in adults (mean age 55 years), RMC is a distinctive entity occurring almost exclusively in young African American men (usually <30 years) with sickle cell trait. Both these tumours are rare but biologically extremely aggressive subtypes of RCC, with the majority of patients presenting with metastatic disease. ${ }^{1,2}$

In recent years, needle biopsies from renal masses have been performed increasingly. ${ }^{3}$ In these small biopsies, the entire range of morphological features necessary to make a diagnosis may not be appreciated fully due to sampling issues. Immunohistochemistry is often helpful in this setting to narrow the differential diagnosis (UC versus RCC) and/or to arrive at a definitive diagnosis. The goal of this study is to evaluate the utility of an optimal immunohistochemical panel to differentiate accurately high-grade, poorly differentiated and infiltrative carcinomas involving the renal sinus region, with emphasis on distinguishing invasive UC of upper urinary tract from high-grade RCCs, including CDC and RMC.

\section{Materials and methods}

CASE SELECTION

After approval from the University of Michigan Institutional Review Board for human subject re- search, tumours were identified via a Systematized Nomenclature of Medicine (SNOMED) search of the pathology database. A total of 64 high-grade renal tumours were identified, including 18 aggressive (pT3 or higher pathological stage) invasive UC involving renal pelvis/upper urinary tract, six CDC, two RMC, 18 type 2 PRCC and 20 CRCC (Fuhrman nuclear grade $>3$ ). All cases included were resection specimens (radical or partial nephrectomy) and all haematoxylin and eosin (H\&E)-stained sections were reviewed by study pathologists (J.C., L.P.K.) for confirmation of the diagnosis according to the World Health Organization (WHO) 2004 criteria. $^{1}$ The invasive high-grade UCs were classified according to the 2004 WHO/International Society of Urological Pathology (ISUP) classification. The cases included in the study were a mixture of usual and diagnostically challenging tumours.

TISSUE MICROARRAY (TMA) CONSTRUCTION AND CLUSTER ANALYSIS

A TMA was constructed from 0.6-mm cores of formalin-fixed, paraffin-embedded neoplastic tissue in triplicate as well as representative normal kidney sections from the same cases for controls. TMA technology is cost-effective and allows for high-throughput immunohistochemical profiling of tumours in a model that simulates small biopsy sampling. Three cores were sampled from each case to account for tumour heterogeneity. The TMA slides were stained with a select panel of 11 antibodies using standard immunohistochemical techniques on an automated Ventana Benchmark XT stainer (Ventana, Phoenix, AZ, USA) or a Dako AutoStainer (Dako, Carpinteria, CA, USA). The list of antibodies, clones, origins, titrations, pretreatments, incubation times and expected staining characteristics are listed in Table 1. The expression of each antibody was characterized on a $0-2$ scale, where 0 represented staining in $0 \%$ to $<10 \%$ of cells, 1 represented focal expression in $10 \%$ to $<50 \%$ of cells and 2 represented diffuse expression in $>50 \%$ of cells with moderate to strong staining intensity. Scores of 1 and 2 were considered to be positive. Immunohistochemical evaluation was performed independently and blindly by two study pathologists (J.C., L.P.K.) with expertise in genitourinary disease. Differences of opinion in rare instances were resolved by consensus evaluation of the case with all pathologists in the study. Immunohistochemical expression results expressed as plain scores $(0,1,2)$ were arranged in a text delimited file and broadcasted from the Data Matrix Viewer module of the gaggle software suite (http:// 
Table 1. List of antibodies, staining patterns and treatment conditions

\begin{tabular}{|c|c|c|c|c|c|}
\hline Antibody & Clone & Staining pattern & Company & Dilution & Pretreatment \\
\hline CK7 & OV-TL 12/30 & Cytoplasmic & Dako & $1: 50$ & Buffer at $\mathrm{pH} 8.0$ (30 min) \\
\hline p63 & $4 \mathrm{~A} 4$ & Nuclear & $\begin{array}{l}\text { Thermo Scientific, Fremont, } \\
\text { CA, USA }\end{array}$ & $1: 200$ & Buffer at $\mathrm{pH} 8.5$ (30 min) \\
\hline Vimentin & V9 & Cytoplasmic & Dako & $1: 400$ & Buffer at $\mathrm{pH} 8.0$ (30 min) \\
\hline PAX8 & Polyclonal & Nuclear & $\begin{array}{l}\text { Proteintech Group, Chicago, } \\
\text { IL, USA }\end{array}$ & $1: 200$ & Buffer at $\mathrm{pH} 6.0$ (15 min) \\
\hline PAX2 & Polyclonal & Nuclear & $\begin{array}{l}\text { Zymed/Invitrogen, Carlsbad, } \\
\text { CA, USA }\end{array}$ & $1: 50$ & Buffer at $\mathrm{pH} 8.0$ (60 min) \\
\hline CD10 & $56 C 6$ & $\begin{array}{l}\text { Cytoplasmic/ } \\
\text { membranous }\end{array}$ & Ventana, Tucson, AZ, USA & Predilute & Buffer at $\mathrm{pH} 8.0$ (60 min) \\
\hline RCC antigen & $\mathrm{PN}-15$ & Membranous & Ventana & Predilute & Protease $1-12 \mathrm{~min}$ \\
\hline CAIX & Polyclonal & Membranous & Abcam, Cambridge, MA, USA & $1: 200$ & Buffer at $\mathrm{pH} 6.0$ (10 min) \\
\hline HMCK & $34 \beta \mathrm{E} 12$ & Cytoplasmic & Dako & $1: 50$ & Buffer at 8.0 (60 min) \\
\hline AMACR (P504S) & $13 \mathrm{H} 4$ & Cytoplasmic & Zeta, Sierra Madre, CA, USA & $1: 40$ & Buffer at $\mathrm{pH} 8.0$ (30 min) \\
\hline S100A1 & Proprietary & $\begin{array}{l}\text { Cytoplasmic } \\
\text { or nuclear }\end{array}$ & $\begin{array}{l}\text { Sigma-Aldrich, St Louis, } \\
M O, \text { USA }\end{array}$ & $1: 50$ & Buffer at $\mathrm{pH} 6.0$ (15 min) \\
\hline $\mathrm{INI}-1$ & MRQ-27 & Nuclear & Cell Marque, Rocklin, CA, USA & Predilute & Buffer at $\mathrm{pH} 8.0$ (60 min) \\
\hline
\end{tabular}

AMACR, Alpha-methylacyl-CoA racemase; CAIX, carbonic anhydrase IX; HMCK, high molecular weight cytokeratin; INI-1, integrase interactor 1; PAX, paired box gene; RCC, renal cell carcinoma; S100A1, S100 calcium-binding protein A1.

gaggle.systemsbiology.org/docs) to the Multi-Experiment viewer of the TM4 software suite (http:// www.tm4.org/mev.html). Unsupervised hierarchical clustering was performed using average linkage analysis with Euclidean distance metric and the data were divided at the second branch point down in the cluster tree. Given the broad panel of antibodies that were analysed, several markers were found to have overlapping staining patterns or were found to have minimal discriminating properties among the different tumour types. After removal of these antibodies, a limited refined panel of markers was included in a final unsupervised cluster analysis. From this cluster plot, the specificity and sensitivity of selected markers was calculated for each tumour types.

\section{Results}

IMMUNOHISTOCHEMICAL STAINING PATTERNS OF EACH TUMOUR TYPE

The results of immunostaining with all 11 antibodies are summarized in Table 2.

\section{Invasive UC}

In contrast to high-grade RCCs, p63 was expressed strongly and diffusely in all UC. In addition, the majority of tumours were diffusely, strongly reactive with both CK7 and HMCK (17 of 18, 94\% each). The majority of UC were negative with PAX8 (15 of 18, $83 \%$ ) and PAX2 (17 of $18,94 \%$ ), although a small subset of UC show predominantly focal reactivity for PAX8 (three of 18, 17\%) and PAX2 (one of 18, 6\%). All UC demonstrate complete lack of reactivity for RCC antigen. Vimentin displayed limited expression in $11 \%$ (two of 18 ) of UC, while variable positivity is seen with CD10, alpha-methylacyl-CoA racemase (AMACR), carbonic anhydrase IX (CAIX) and S100 calcium-binding protein A1 (S100A1). Integrase interactor 1 (INI-1) was positive in all (100\%) cases with strong, diffuse expression. See Figure 3 and Table 2 for details.

CDC

All the CDCs demonstrated an absolute lack of reactivity with p63 (none of six, 100\%). A majority of tumours showed diffuse, strong expression of PAX8 
Table 2. Immunohistochemical profile of urothelial carcinoma and it morphological mimics

\begin{tabular}{|c|c|c|c|c|c|c|c|c|c|c|c|c|}
\hline & \multicolumn{12}{|c|}{ Percentage of cases staining positive (\%) } \\
\hline & P63 & CK7 & Vimentin & INI-1 & PAX-8 & PAX-2 & HMCK & $\mathrm{RCC}$ & CD10 & AMACR & CAIX & S100A1 \\
\hline UC $(n=18)$ & 100 & 94 & 11 & 100 & 17 & 6 & 94 & 0 & 50 & 39 & 33 & 78 \\
\hline $\operatorname{CDC}(n=6)$ & 0 & 50 & 33 & 100 & 83 & 50 & 33 & 17 & 0 & 33 & 17 & 100 \\
\hline $\mathrm{RMC}(n=2)$ & 0 & 100 & 0 & 0 & 100 & 100 & 50 & 0 & 0 & 0 & 0 & 100 \\
\hline $\operatorname{PRCC}(n=18)$ & 0 & 28 & 94 & 100 & 78 & 56 & 0 & 50 & 67 & 94 & 44 & 89 \\
\hline $\operatorname{CRCC}(n=20)$ & 0 & 20 & 90 & 100 & 95 & 70 & 0 & 60 & 95 & 30 & 95 & 85 \\
\hline
\end{tabular}

AMACR, Alpha-methylacyl-CoA racemase; CAIX, carbonic anhydrase IX; CDC, carcinoma of collecting ducts of Bellini; CRCC, clear cell renal cell carcinoma; HMCK, high molecular weight cytokeratin; INI-1, integrase interactor 1; PAX, paired box gene; PRCC, capillary renal cell carcinoma; RCC, renal cell carcinoma; RMC, renal medullary carcinoma; S100A1, S100 calciumbinding protein $A 1$; UC, urothelial carcinoma.

(five of six, 83\%) with a decreased sensitivity for PAX2 (three of six, 50\%) and near-absent expression for RCC antigen (one of six, $17 \%$ ). CK7 was strongly positive in half of CDCs while vimentin was expressed in 33\% (two of six) of tumours. Most of the other markers in the panel, including HMCK, CD10, AMACR and CAIX, were insensitive for CDC. All CDCs expressed S100A1 strongly and diffusely. Interestingly, while the majority (66\%) of CDC showed strong diffuse INI-1 expression, $33 \%$ (two of six) cases showed focal, weak expression. See Table 2 for details.

\section{RMC}

Both cases of RMC were strongly, diffusely positive with CK7, S100A1 and PAX 8, negative with p63, INI-1, vimentin, CA 1X, RCC antigen and AMACR and variably positive with PAX2 and HMCK.

\section{CCRC}

Although not specific, several markers were highly sensitive for CRCC, including vimentin, PAX8, CD10 and CAIX, with strong diffuse expression in $>90 \%$ of the tumour for each antibody. In contrast, no CRCC were positive for p63 or HMCK. PAX2 reactivity was seen in $70 \%$ of CRCC. While PAX2 was less sensitive than PAX8 $(70 \%$ versus $95 \%$ tumours positive, respectively), it was more sensitive than RCC antigen (12 of 20,60\%). The least sensitive antibodies were AMACR (six of 20,30\%) and CK7 (four of 20, 20\%) which mainly demonstrated focal expression. Finally, S100A1 and INI-1 showed strong, diffuse expression in the majority ( $85 \%$ and $95 \%$, respectively) of cases.

\section{PRCC}

Among RCC subtypes, PRCC was the least sensitive for renal markers PAX8 (78\%) and PAX2 (56\%), with only half of tumours reactive with RCC antigen. The most sensitive markers for PRCC are vimentin and AMACR, with reactivity in $94 \%$ of tumours. Similar to CRCC, no PRCC demonstrate reactivity with p63 or HWCK. INI-1 was positive in all cases $(100 \%)$ with strong, diffuse expression in majority ( 16 of $18,89 \%$ ) of cases. The other markers in the panel, CK7, CD10, CAIX and S100A1, had variable staining patterns ranging from $28 \%$ to $89 \%$, respectively. See Figure 1 and Table 2 for details.

\section{CLUSTER ANALYSIS}

An initial unsupervised cluster plot of the full panel of 11 antibodies segregated almost all the RCCs (45 of $46,98 \%)$ from all the UCs based on lack of expression of p63 in all (100\%) RCCs, predominant strong expression of PAX8 and vimentin, predominant lack of expression with CK7 and HMCK and variable expression of RCC antigen, CD10, CAIX and PAX2. The tumours clustered into four distinct subgroups (designated A-D) based on staining similarities, as shown in Figure 1.

Group A is composed predominantly of CRCC (18 of $21,86 \%$ ), with a smaller number of PRCC (three of 21 , $14 \%$ ) and both tumour types demonstrate expression of S100A1, CD10, CAIX, vimentin and INI-1 and similar patterns of reactivity with PAX2 and PAX8. Most of the tumours are negative for CK7 and all the tumours are negative with p63 and HMCK. The three PRCC tumours that segregated into group A were included because of intense staining with CAIX. The majority of CRCC (18 of 20, 90\%) segregated into this cluster.

Group B is populated mainly by PRCC (15 of 24, $63 \%$ ), a subset of CDC (three of six) as well as two CRCC. As a cluster, these tumours are defined by 


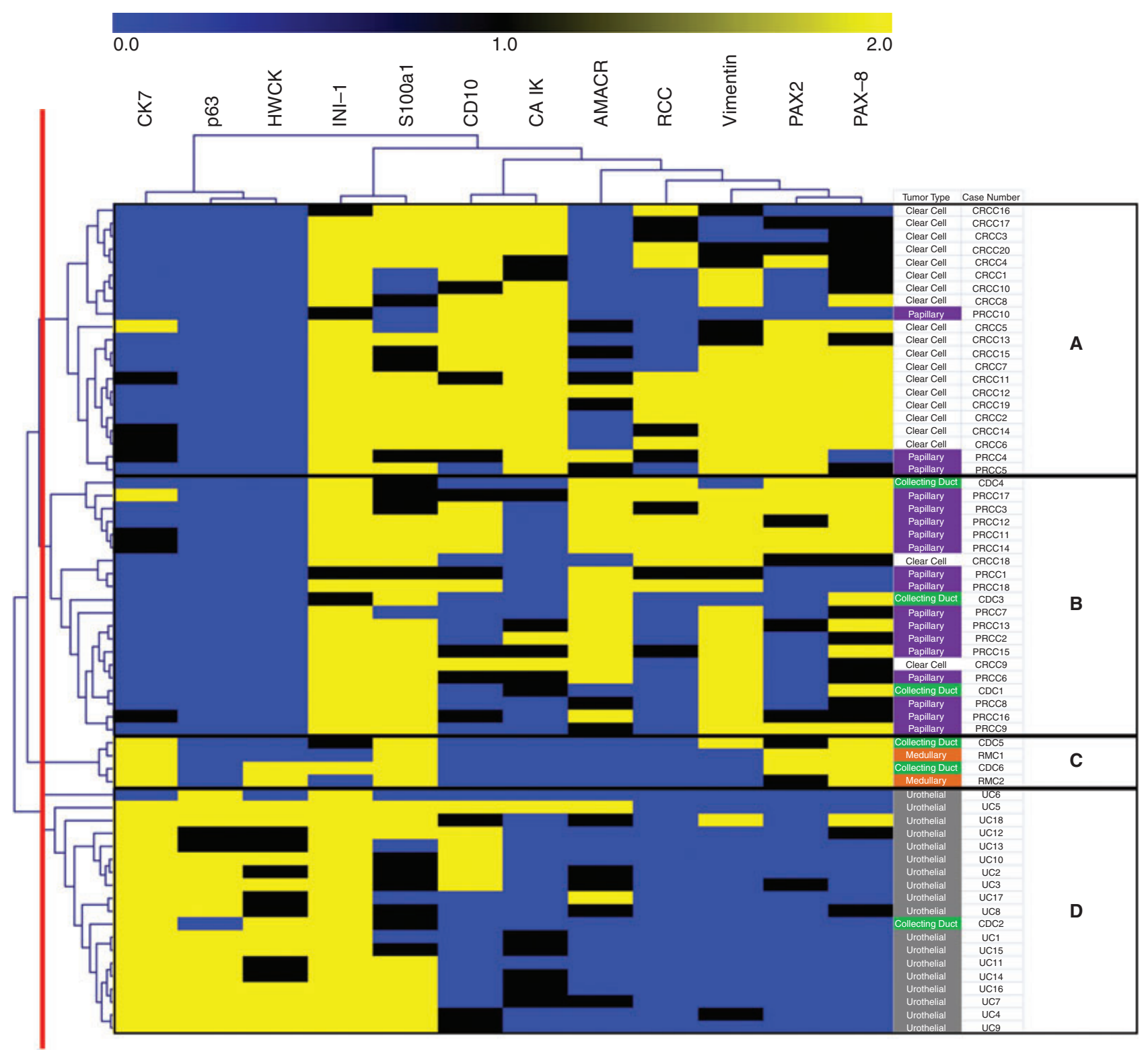

Figure 1. Initial unsupervised cluster plot of the full immunohistochemical panel. Antibodies are arrayed at the top of the map and the four types of tumours are listed along the right side. The red line on the left is the level of the clustering tree that separates the tumours into four groups. Almost all the renal cell carcinomas (RCCs) segregate from all the urothelial carcinomas (UCs) based on lack of expression of p63 in all (100\%) RCCs, predominant strong expression of paired box gene 8 (PAX8) and vimentin, predominant lack of expression with CK7 and high molecular weight cytokeratin (HMCK) and variable expression of RCC, CD10, carbonic anhydrase IX (CAIX) and PAX2. The tumours clustered into four distinct subgroups based on staining similarities. Group A illustrates those tumours driven by expression of renal markers CD10, PAX2, PAX8, integrase interactor 1 (INI-1) and positivity with CAIX but lack of CK7, p63 and HMCK, which includes nearly all clear cell renal cell carcinomas (CRCCs). Group B is composed of a mix of papillary renal cell carcinoma (PRCC) and half the carcinoma of collecting ducts of Bellini (CDC) with similar expression of PAX8, PAX2, vimentin and INI-1 but decreased expression of CD10 and CAIX, increased expression of alpha-methylacyl-CoA racemase (AMACR) and lack of p63 and HMCK. Group C is composed of tumours with similar expressions of CK7, PAX8 and S100 calcium-binding protein A1 (S100A1), PAX8 reactivity stronger than PAX2, lack of expression with p63, CD10, CA1X and variable expression with HMCK and INI-1 and includes both renal medullary carcinomas (RMCs) and a subset of CDC. Both RMCs lack INI-1, vimentin and p63 expression and are strongly positive with CK7 and PAX8. Finally, group D is populated almost exclusively by UC, which lack expression of renal markers and have intense reactivity with CK7, p63 and high molecular weight kininogen (HWCK). The only CDC lacking expression of renal markers segregated to group D. 


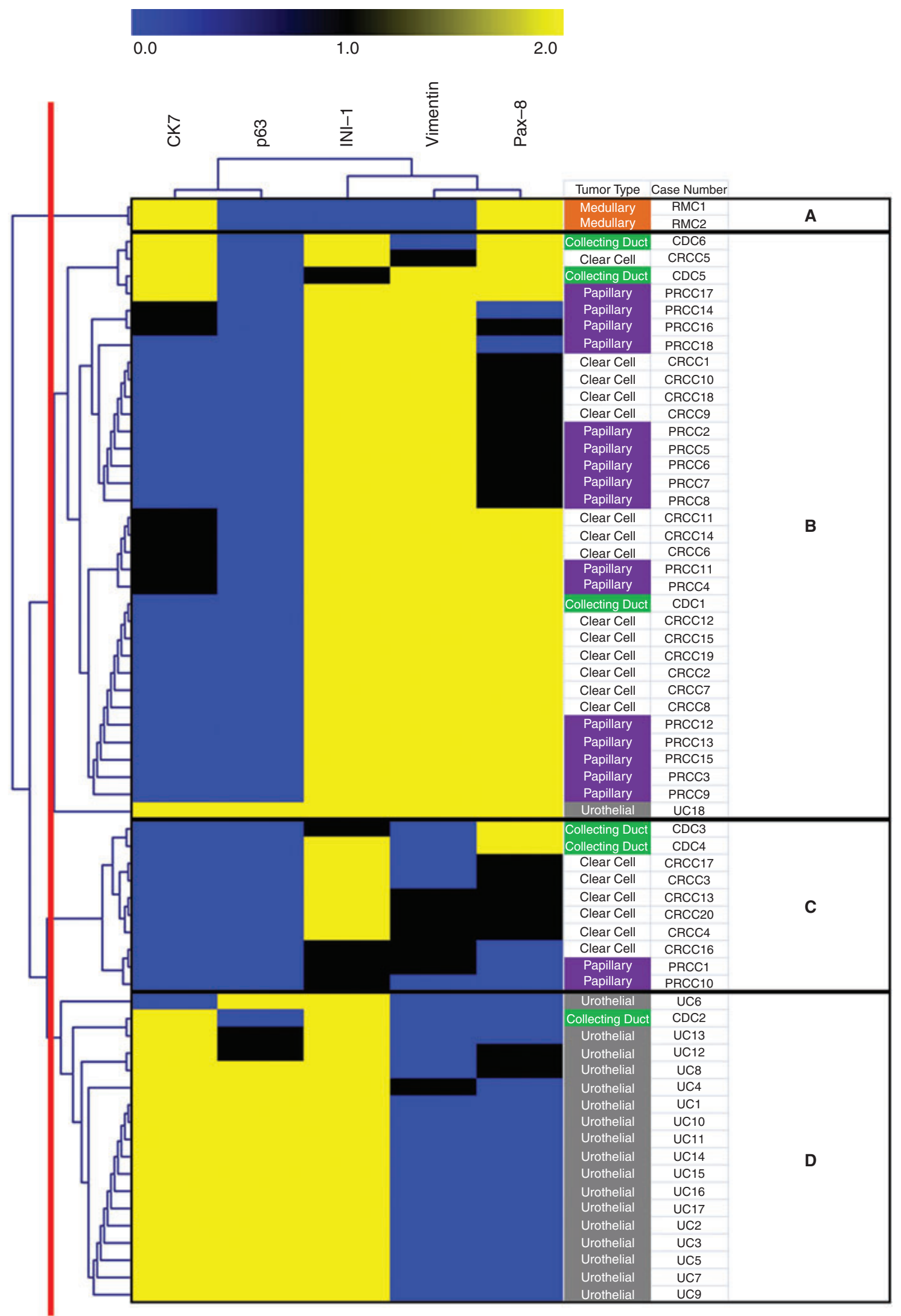

(C) 2012 Blackwell Publishing Ltd, Histopathology, 60, 597-608. 
expression of S100A1, INI-1, vimentin, AMACR, predominant lack of CK7 expression, PAX8 reactivity greater than PAX2 and no expression of p63 or HMCK. The subset of CDC (three of six, 50\%) included in this cluster is characterized by lack of expression with CK7 and HMCK staining, strong PAX8 and INI-1 expression and variable expression with vimentin. The majority of PRCC (15 of $18,83 \%)$ segregated into cluster B.

Group C is composed of both RMCs and a subset of CDC (two of six, 33\%), which as a cluster are defined by intense staining with CK7, PAX8 and S100A1 and PAX8 reactivity stronger than PAX2, lack of expression with p63, CD10, CA1X and variable expression with HMCK and INI-1. The two CDCs included in this group are characterized by strong expression of CK7 and PAX8, positive INI-1, variable expression of vimentin and lack of staining with p63. Both RMCs lack INI-1, vimentin and p63 expression and are strongly positive with CK7 and PAX8.

All the UCs (18 of $18,100 \%)$ and a single CDC segregated into group $\mathrm{D}$, which is characterized by intense staining with CK7, p63, HMCK and INI-1, variable expression of S100A1 and a predominant lack of reactivity with renal markers RCC antigen, vimentin, PAX2 and PAX8. The single CDC that segregated in this group was negative with p63, PAX2 and PAX8.

Several overlapping expression patterns were delineated by the initial unsupervised cluster analysis of the panel of antibodies examined. Three sets of antibodies (CD10 and CAIX, PAX2 and PAX8 and HMWK and p63) (see Table 2) clustered together among the four tumour types (see Figure 1).

The initial unsupervised cluster plot (Figure 1) along with expression percentages of the 11 antibodies for four tumour types was reviewed and antibodies with significant overlap or minimal impact in distinguishing between the four types of renal tumours were removed. An unsupervised cluster analysis with a select and limited panel that included p63, CK7, vimentin, INI-1 and PAX8 was performed (see Figure 2).
The cluster plot of the select antibody panel separated all the cases into four distinct groups at the third division point of the cluster tree.

Cluster A is composed exclusively of both RMCs characterized by strong expression of CK7 and PAX8 and lack of staining with p63, INI-1 and vimentin. Clusters B and C are composed of a mixture of CRCC, PRCC and CDC, but have different staining characteristics. The 10 tumours segregating into cluster C, composed predominantly of CRCC (six cases, 60\%), a subset of PRCC (two cases, 20\%) and CDCs (two cases, $20 \%$ ), are completely negative for CK7 and p63, show predominantly strong INI-1 expression, have focal to absent staining with vimentin and variable staining with PAX8. In comparison, cluster B, composed of a majority of CRCC and PRCC $(75 \%$ and $89 \%$, respectively), a subset (50\%) of CDC and a single case of UC, is characterized by intense diffuse staining with vimentin and INI-1, PAX8 positivity in the majority of cases, CK 7 expression in a subset of cases and no p63 expression in all RCCs. The single UC in this cluster is positive for p63 and segregated into this group based on strong reactivity with both PAX8 and vimentin.

Finally, group D includes the majority of the UC (17 of $18,94 \%$ ) as well as one CDC, and is characterized predominantly by intense and diffuse staining with CK7, p63 and INI-1 and lacks vimentin and PAX8 expression. The single CDC case in this cluster is negative with both PAX 8 and p63.

\section{SENSITIVITY, SPECIFICITY AND POSITIVE PREDICTIVE VALUES}

The sensitivity and specificity of the antibodies that comprise our panel for distinguishing collecting duct carcinoma from its morphological mimics is presented in Table 3.

P63 expression in UC was found to be both sensitive and specific (100\% and $100 \%$, respectively) in distinguishing UC from all RCC subtypes. Lack of INI-1

\footnotetext{
Figure 2. Unsupervised cluster map of optimal panel of markers with urothelial carcinoma (UC), carcinoma of collecting ducts of Bellini (CDC), renal medullary carcinoma (RMC), clear cell renal cell carcinoma (CRCC) and papillary renal cell carcinoma (PRCC). Antibodies are arrayed at the top of the map and the various types of tumours are along the right side. The red line on the left is the level of the clustering tree that separates the tumours into four groups. Group A, composed exclusively of both RMCs, is characterized by strong expression of CK7 and paired box gene 8 (PAX8) and lack of staining with p63, integrase interactor 1 (INI-1) and vimentin. Groups B and C are composed of a mixture of CRCC, PRCC and CDC but have different staining characteristics. Group B, composed of a majority of CRCC and PRCC, a subset of CDC and a single case of UC, is characterized by intense diffuse staining with vimentin and INI-1, PAX8 positivity in the majority of cases, CK7 expression in a subset of cases and no p63 expression in all renal cell carcinomas (RCCs). The single case of UC, which is positive for p63, segregated into this group based on strong reactivity with both PAX8 and vimentin. Group C, which is defined by a lack of p63 and CK7, predominantly diffuse reactivity with INI-1 and variable expression of vimetin, and PAX8, is populated by a subset of CRCC, PRCC and CDC. Finally, cluster D is nearly all UC, with the only CDC not expressing PAX8. The tumours in this cluster are defined by intense reactivity with CK7, p63 and INI-1 with lack of staining for vimentin and PAX8.
} 
expression in RMC was both sensitive and specific (100\% and 100\%, respectively) in distinguishing RMC from UC and other subtypes of RCC. A combined panel of p63, PAX8 and IN1-1 decreases sensitivity of UC detection to $83 \%$ due to a small number of UC that are positive for PAX8, but specificity remains at $100 \%$. In distinguishing CRCC and PRCC from UC, RMC and CDC, positive reactivity with vimentin was found to have a sensitivity of $89 \%$ and specificity of $88 \%$. With the addition of CK7 to vimentin, the sensitivity and specificity drop to $73 \%$ and $68 \%$, respectively. No unique profile could separate CRCC from PRCC.

Utilizing the panel of select antibodies based on our cluster analysis, the positive predictive value (PPV) for the diagnosis of UC (p63+) compared to the four subtypes of RCC with only p63 is $100 \%$. When distinguishing UC (p63+/PAX8-/INI-1+) from CDC (p63-/PAX8+/INI-1+) or RMC (p63-/PAX8+/INI$1-)$, the PPV is $95 \%$. The PPV of a tumour not being a PRCC or CRCC with absent staining for vimentin is $89 \%$. When positive reactivity for CK7 is added, the PPV decreases to 70\%. Lastly, in differentiating CDC and RMC from CRCC and PRCC based on absence of vimentin positivity, the PPV is $89 \%$.

\section{Discussion}

The diagnosis of poorly differentiated, high-grade carcinomas involving the renal sinus region is often problematic. High-grade UCs of the upper urinary tract frequently present with infiltrative masses that sometimes extensively involve the renal parenchyma mimicking RCCs. UC with glandular features shows frequent morphological overlap with RCC, notably $\mathrm{CDC}$ and RMC, both of which are rare but aggressive subtypes of RCC. The major criteria for diagnosis of CDC include epicentre of tumour in the renal medulla as well as exclusion of UC involving the upper tract. ${ }^{1}$ Immunohistochemical reactivity of CDC with HMCK and Ulex europaeus agglutinin lectin have been included among major criteria for diagnosis of $\mathrm{CDC}$; however, they have limited utility, as both UC and CDC are positive with these markers. ${ }^{1,4}$ We have evaluated an expanded panel of immunohistochemical markers including recent novel markers with the goal of developing a select optimal panel that can distinguish high-grade, poorly differentiated and infiltrative carcinomas involving the renal sinus region, with emphasis on distinguishing invasive UCs of upper urinary tract from high-grade RCCs, including CDC and RMC.

Our approach of cluster tree analysis to high-volume immunohistochemical data ${ }^{5}$ confirms previous studies that many markers (S100A1, CD10, AMACR and CAIX) have overlapping staining patterns, limiting their utility in day-to-day practice. We found that a select panel of CK7, p63, vimentin, PAX8 and INI-1 offers the greatest sensitivity, specificity and predictive value and defined our select optimal panel for sorting out this differential diagnosis (see Figures 2 and 3).

Our results, using a broad panel as seen in the initial unsupervised analysis (Figure 1), show almost all the RCCs (45 of 46, 98\%) segregating from UCs as a result of lack of p63 expression, diffuse expression of vimentin and variable expression of CK7, PAX 8, PAX 2 and RCC antigen. In comparison, all the UC demonstrated strong diffuse expression of p63 and the majority of UC (17 of $18,94 \%$ ) demonstrate strong expression of CK7. The single case of CDC which segregated with all the UC was negative with p63, PAX2 and PAX8. Thus, in our cohort, strong, diffuse expression of p63 in UC was

Table 3. Sensitivity and specificity of antibodies that comprise the optimal panel in the differential diagnosis of urothelial carcinoma and it morphological mimics

\begin{tabular}{llll}
\hline Differential diagnoses & Panel markers & Sensitivity (\%) & Specificity (\%) \\
\hline UC versus all RCC subtypes & p63 (+) & 100 & 100 \\
\hline UC versus RMC & INI-1 (+) & 100 & 100 \\
\hline UC versus CDC or RMC & p63 (+), INI-1 (+) and PAX8 (-) & 83 & 100 \\
\hline$U C$, CDC, RMC versus PRCC, CRCC & Vimentin (-) & 89 & 88 \\
\hline UC, CDC, RMC versus PRCC, CRCC & Vimentin (-) and CK7 (+) & 73 & 68 \\
\hline CDC, RMC versus PRCC, CRCC & Vimentin (-) & 75 & 92
\end{tabular}

CDC, Carcinoma of collecting ducts of Bellini; CRCC, clear cell renal cell carcinomas; INI-1, integrase interactor 1; PAX8, paired box gene 8; PRCC, papillary renal cell carcinomas; RCC, renal cell carcinoma; RMC, renal medullary carcinomas; UC, urothelial carcinoma. 
most valuable in distinguishing UC from RCC including CDC and RMC.

Our expanded panel also included S100A1, AMACR, CAIX and CD10, of which our results of S100A1, CD10 and AMACR are within the ranges of previous studies ${ }^{6-}$ 10 (see Table 2). Interestingly, we found S100A1 to be expressed by majority of UC and RCC and hence this marker has limited utility in differentiating between these tumours. The staining characteristics of CAIX were slightly different from those reported previously did not prove to be useful enough to be included in our select panel. Recently, Gupta et al. ${ }^{11}$ examined CAIX in renal epithelial neoplasms and found CAIX to be helpful in differentiating between $\mathrm{CDC}$ and UC, as expression of CAIX was present in the majority of UC. While the CAIX expression in type 2 PRCC and CRCC in our study was similar to Gupta et al., we found CAIX expression in only a subset of UC (33\%), rendering it of limited utility in distinguishing it from CDC. Hence, these immunohistochemical markers (S100A1, AMACR, CAIX and CD10) did not add any discriminating power and were of no significant utility.

PAX2 and PAX8 are members of the PAX gene transcription factors family. PAX8, a more recently described marker, is essential for thyroid, metanephron and Müllerian duct lineage commitment, $^{12}$ while PAX2 is essential for development of kidney during fetal life. ${ }^{11}$ PAX8 is strongly positive (nuclear expression) in normal kidney and is expressed by collecting ducts and differentiating nephrons. PAX2 has emerged as a relatively sensitive and specific marker of RCC with variable expression in the different subtypes of RCCs. ${ }^{10}$ PAX2 expression is noted in the majority of clear cell RCCs and type 1 PRCCs; however, some subtypes of RCC, including chromophobe RCC, tubulocystic RCC and translocation-associated RCCs, show decreased sensitivity with PAX2. Previous studies have reported conflicting results regarding PAX2 expression in CDC; Gupta et al. ${ }^{11}$ found no PAX2 expression in CDC (five cases evaluated), while Ozcan et al. ${ }^{13}$ found all cases to be positive with PAX2 (five cases evaluated). In our study, PAX2 expression was noted in 50\% (three of six) of CDC. PAX2 was also positive in both cases of RMC with focal staining in one case. PAX8 is emerging as a specific markers for RCC, ${ }^{14}$ expressed by a majority of RCCs. ${ }^{12,14}$ One of the interesting observations in our study is the finding that PAX8 is overall a more sensitive marker for the detection of RCCs, including CDC compared to PAX2 (83\% versus 50\%, respectively), and hence was included in our select panel. Of all the high-grade RCCs in our cohort, $2 \%$ (one of 46 , one PRCC) cases were positive with PAX2 and negative with PAX8, while 26\% (12 of 46) were negative with
PAX2 and positive with PAX8. Overall, $11 \%$ of all RCCs (five of 46, two CRCC, two PRCC and one CDC) were negative with both markers. Our recent study, ${ }^{15}$ comparing utility of PAX8 and PAX2 in diagnosis of RCC in cytology specimens, also showed similar findings, with PAX8 showing slightly higher sensitivity compared to PAX2 (88\% versus $83 \%$, respectively). RCC antigen was excluded from our select panel because of significantly decreased sensitivity compared to PAX8 and PAX2.

HMCK and p63 show similar expression profiles, with strong, diffuse expression in a majority of UC and lack of reactivity in type 2 PRCC and CRCC. CDC, RMC and UC show overlapping expression patterns with HMCK, as almost a third of CDC and half of RMC in our cohort are positive with this marker, confirming previous studies; ${ }^{10,16}$ however, no p63 expression was recognized in any CDC or RMC. The results of our study support the utility of p63 as a sensitive and specific marker of UC with diffuse nuclear expression. ${ }^{17,18} \mathrm{UC}, \mathrm{CDC}$ and RMC also show overlapping staining patterns with CK7 and vimentin, with the majority of these tumours showing predominantly positive CK7 reactivity and negative expression with vimentin. This is distinct from CRCC and type 2 PRCCs, which tend to be predominantly negative with CK7 and HMCK and are positive with vimentin. These findings are in agreement with previous studies. ${ }^{10}$ In our experience, the predominant utility of CK7 and vimentin is to distinguish RMC, CDC and UC from type 2 PRCC and CRCC. The immunoprofile of CK7 (+)/vimentin (-) supports a diagnosis of RMC or CDC or UC (sensitivity $73 \%$ and specificity $68 \%$ ). Interestingly, in our cohort, vimentin alone could differentiate RMC and CDC from PRCC and CRCC (sensitivity of $75 \%$, specificity of $92 \%$ ), yet the unsupervised cluster analysis was not able to differentiate sufficiently between type 2 PRCC and CRCC. See Table 3 for details.

After reviewing the initial unsupervised cluster analysis and excluding markers with limited discriminating powers (S100A1, AMACR, CD10 and CAIX), low sensitivity (PAX2 had lower sensitivity compared to PAX8 but was more sensitive compared to RCC antigen) and/or overlapping staining patterns compared to other similar markers (RCC antigen, PAX2, HMCK), a second unsupervised cluster analysis was performed using a select panel composed of CK7, p63, INI-1, vimentin and PAX8 (see Figure 3).

A panel of PAX8, p63 and INI-1 is optimal in distinguishing UC from $\mathrm{CDC}$ and RMC as all three tumours commonly show similar staining patterns with CK7 and vimentin. PAX8 is a sensitive marker of CDC and stains the majority of $\mathrm{CDC}(83 \%)$ and both 
RMCs in our cohort. It is more sensitive compared to PAX 2, which only stained 50\% of CDC and was focally positive in one RMC. This finding is fully in keeping with a recent study that analysed a large series of CDC (21 cases) and found all CDC to be positive with PAX8. The majority of UC (83\%) involving the upper tract in our study were negative for PAX8; a finding confirmed by other studies $(77-91 \%) .{ }^{12,19}$ Our experience with p63 in UC involving upper urinary tract supports a previous study ${ }^{18}$ that has shown p63 to be useful in distinguishing UC from high-grade RCC, including CDC and RMC (100\% specificity for UC with no staining of any RCC with p63). In our study, p63 was the single most useful marker in the distinction of UC from RCC, as no high-grade RCC in our cohort, including CDC and RMC, showed reactivity with p63. INI-1 is most useful in the distinction of RMC from UC and other subtypes of
RCC. Loss of the INI-1 tumour suppressor gene has been shown in paediatric rhabdoid tumours of kidney. A recent study ${ }^{20}$ demonstrated loss of heterozygosity (LOH) of the hSNF 5/INI gene and a corresponding lack of INI-1 immunoreactivity in RMCs. We analysed INI-1 expression in our cohort of cases and, as expected, found strong, diffuse INI-1 expression in the vast majority of UC, CDC CRCC and type 2 PRCC. As expected, complete loss of INI-1 expression was observed in both cases of RMCs. Interestingly, focal, weak expression of INI-1 was observed in a subset of CDC (two of six, 33\%), while the remaining cases showed diffuse, moderate to strong INI-1 expression. While we did not observe any CDC case with complete loss of INI-1 expression, a recent unpublished abstract $^{21}$ found INI-1 expression to be lost $(20 \%)$ or minimally expressed $(10 \%)$ in a subset of CDC. These

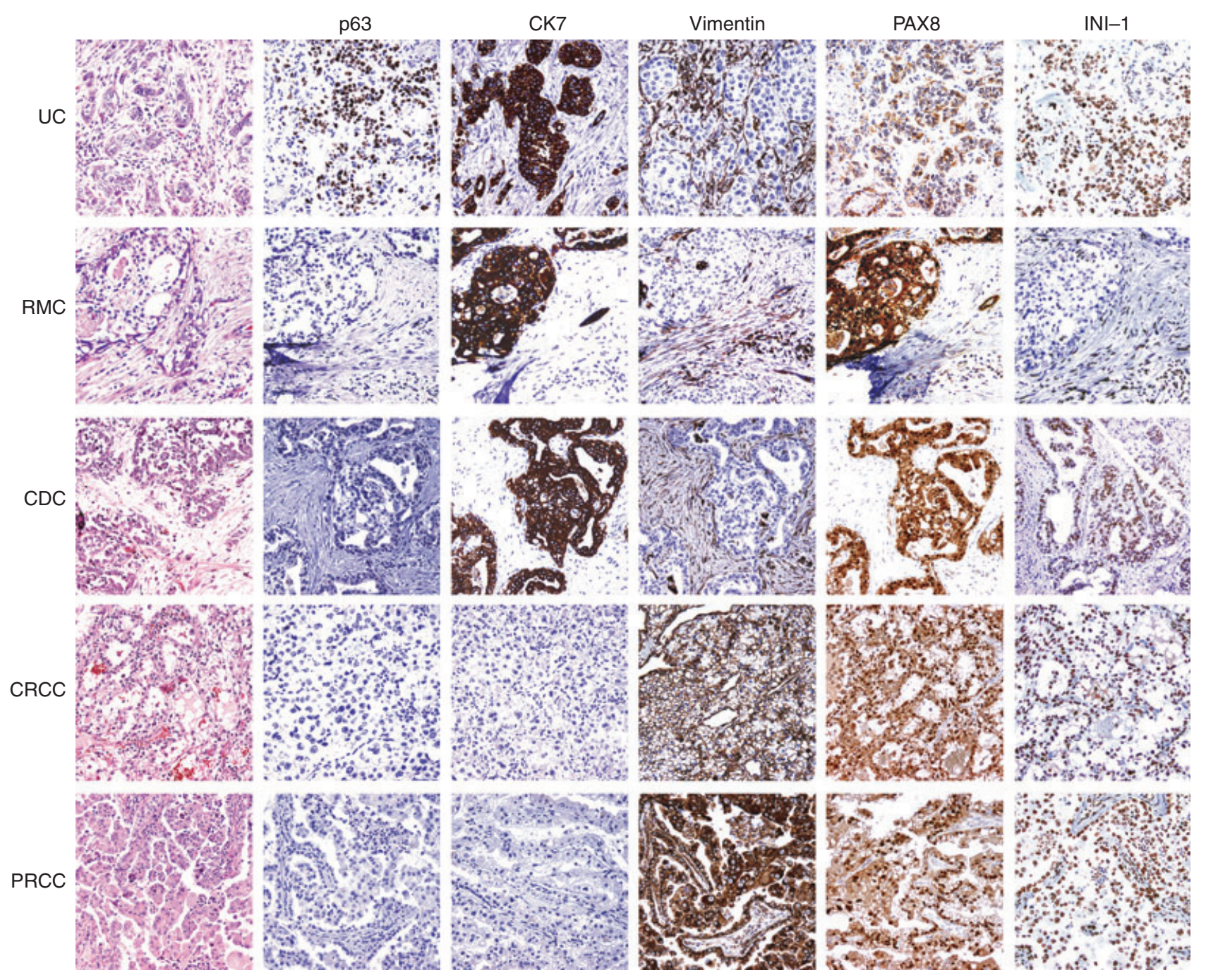

Figure 3. Representative examples of the tumours involving the renal hilum and their staining patterns as shown with the select optimal panel of antibodies comprising of CK7, p63, vimentin, paired box gene 8 (PAX8) and integrase interactor 1 (INI-1). 
findings, which suggest that at least a subset of CDCs may be related to RMCs, can be explored with molecular studies.

An immunoprofile of PAX8 (-)/p63 (+)/INI-1 (+) supports the diagnosis of UC involving upper urinary tract (sensitivity 83\%, specificity 100\%), a PAX8 $(+) /$ p63 (-)/INI-1(+) immunoprofile supports a diagnosis of RCC and favours CDC (sensitivity $88 \%$, specificity 100\%), while an immunoprofile of PAX8 (+)/p63 $(-) / \mathrm{INI}-1(-)$ supports a diagnosis of RMC in the correct clinical setting (sensitivity 100\%, specificity 100\%). The study by Albadine et al. ${ }^{19}$ found p63 positivity in a small subset (three of $21,14 \%$ ) of CDC, a finding not noted in our cohort of CDCs. Thus, in the unusual scenario of PAX8 (+)/p63 (+) immunoprofile, IHC alone may not be able to distinguish UC definitively from CDC, as both tumours are positive with INI-1. In these cases, additional clinical information including positive urine cytology, the presence of urothelial carcinoma in situ along the renal pelvis, etc. may be useful features to support a diagnosis of UC. A recent unpublished abstract, $^{22}$ which analysed 11 cases of RMC, found PAX8 and p63 positivity in $100 \%$ and $58 \%$, respectively, and postulated that PAX8+/p63+ immunoprofile supports the diagnosis with a sensitivity of $58 \%$ and specificity of $89 \%$. However, both cases of RMC in our cohort showed an immunoprofile of PAX8 (+)/p63 (-), similar to other RCCs, including CDC, but lacked INI-1 expression, unlike other RCC subtypes and UC.

There are some limitations to our study. RMC, an extremely uncommon but high-grade RCC arising in the renal medulla and occurring almost exclusively in young African American male patients with sickle cell trait, has not been represented extensively in our study. Another somewhat smaller drawback of our study is the lack of inclusion of Ulex europaeus lectin agglutinin in our expanded panel. This is not a significant limitation, as previous studies ${ }^{9}$ have confirmed that this marker is expressed by both UC and CDC and therefore is not effective in distinguishing these entities.

In summary, we found a select panel of p63, CK7, vimentin, PAX8 and INI-1 to be most useful in distinguishing between high-grade carcinomas (UC, CDC, RMC, type 2 PRCC and CRCC) involving the renal sinus region. p63 is most useful in distinguishing UC and its positivity separates all UC from RCC mimics including CDC and RMC. UC, CDC and RMC can have overlapping immunohistochemical profiles, but can be distinguished by intense p63 expression in UC and lack of INI-1 expression in RMC. INI-1 expression is exclusively lost in RMC and is expressed minimally in a subset of CDCs, an interesting finding that requires confirmation with molecular studies. PAX8 is also helpful in separating
UC, which is rarely reactive, from high-grade RCCs involving the renal sinus region $(\mathrm{CDC}, \mathrm{RMC}$, type 2 PRCC and CRCC) which demonstrate increased levels of expression. In addition, the sensitivity of PAX8 is significantly greater compared to PAX2 for all RCC subtypes. S100A1 is expressed by the majority of UC and RCC and is not helpful in distinguishing UC from RCC. Using the antibodies tested here, we did not identify a unique immunoprofile differentiating PRCC from CRCC.

\section{Acknowledgement}

The authors would like to thank Javed Siddiqui for assistance in tissue microarray construction.

\section{References}

1. Eble JNSG, Epstein JI, Sesterhenn IA eds. World Health Organization classification of tumours. Lyon: IARC Press, 2004.

2. Tokuda N, Naito S, Matsuzaki O, Nagashima Y, Ozono S, Igarashi T. Collecting duct (Bellini duct) renal cell carcinoma: a nationwide survey in Japan. J. Urol. 2006; 176; 40-43; discussion 43.

3. Shah RB, Bakshi N, Hafez KS, Wood D Jr, Kunju LP. Imageguided biopsy in the evaluation of renal mass lesions in contemporary urological practice: indications, adequacy, clinical impact, and limitations of the pathological diagnosis. Hum. Pathol. 2005; 36; 1309-1315.

4. Srigley JR, Eble JN. Collecting duct carcinoma of kidney. Semin. Diagn. Pathol. 1998; 15; 54-67.

5. Carvalho JC, Wasco MJ, Kunju LP, Thomas DG, Shah RB. Cluster analysis of immunohistochemical profiles delineates CK7, vimentin, S100A1 and C-kit (CD117) as an optimal panel in the differential diagnosis of renal oncocytoma from its mimics. Histopathology 2011; 58; 169-179.

6. Yao R, Lopez-Beltran A, Maclennan GT, Montironi R, Eble JN, Cheng L. Expression of $\mathrm{S} 100$ protein family members in the pathogenesis of bladder tumors. Anticancer Res. 2007; 27; 30513058.

7. Rocca PC, Brunelli M, Gobbo S et al. Diagnostic utility of S100A1 expression in renal cell neoplasms: an immunohistochemical and quantitative RT-PCR study. Mod. Pathol. 2007; 20; 722-728.

8. Molinie V, Balaton A, Rotman S et al. Alpha-methyl CoA racemase expression in renal cell carcinomas. Hum. Pathol. 2006; 37; 698-703.

9. Tretiakova MS, Sahoo S, Takahashi M et al. Expression of alphamethylacyl-CoA racemase in papillary renal cell carcinoma. Am. J. Surg. Pathol. 2004; 28; 69-76.

10. Kobayashi N, Matsuzaki O, Shirai S, Aoki I, Yao M, Nagashima Y. Collecting duct carcinoma of the kidney: an immunohistochemical evaluation of the use of antibodies for differential diagnosis. Hum. Pathol. 2008; 39; 1350-1359.

11. Gupta R, Balzer B, Picken M et al. Diagnostic implications of transcription factor Pax 2 protein and transmembrane enzyme complex carbonic anhydrase IX immunoreactivity in adult renal epithelial neoplasms. Am. J. Surg. Pathol. 2009; 33; 241-247.

12. Tong GX, Yu WM, Beaubier NT et al. Expression of PAX8 in normal and neoplastic renal tissues: an immunohistochemical study. Mod. Pathol. 2009; 22; 1218-1227.

13. Ozcan A, Zhai Q, Javed R et al. PAX-2 is a helpful marker for diagnosing metastatic renal cell carcinoma: comparison with the 
renal cell carcinoma marker antigen and kidney-specific cadherin. Arch. Pathol. Lab. Med. 2010; 134; 1121-1129.

14. Ozcan A, Shen SS, Hamilton C et al. PAX 8 expression in nonneoplastic tissues, primary tumors, and metastatic tumors: a comprehensive immunohistochemical study. Mod. Pathol. 2011; 24; 751-764.

15. Knoepp SM, Kunju LP, Roh MH. Utility of PAX8 and PAX2 immunohistochemistry in the identification of renal cell carcinoma in diagnostic cytology. Diagn. Cytopathol. 2010, [Epub ahead of print] DOI: $10.1002 /$ dc 21590.

16. Gupta R, Paner GP, Amin MB. Neoplasms of the upper urinary tract: a review with focus on urothelial carcinoma of the pelvicalyceal system and aspects related to its diagnosis and reporting. Adv. Anat. Pathol. 2008; 15; 127-139.

17. Kunju LP, Mehra R, Snyder M, Shah RB. Prostate-specific antigen, high-molecular-weight cytokeratin (clone 34betaE12), and/or p63: an optimal immunohistochemical panel to distinguish poorly differentiated prostate adenocarcinoma from urothelial carcinoma. Am. J. Clin. Pathol. 2006; 125; 675681.
18. Langner C, Ratschek M, Tsybrovskyy O, Schips L, Zigeuner R. P63 immunoreactivity distinguishes upper urinary tract transitionalcell carcinoma and renal-cell carcinoma even in poorly differentiated tumors. J. Histochem. Cytochem. 2003; 51; 1097-1099.

19. Albadine R, Schultz L, Illei P et al. PAX8(+)/p63(-) immunostaining pattern in renal collecting duct carcinoma (CDC): a useful immunoprofile in the differential diagnosis of CDC versus urothelial carcinoma of upper urinary tract. Am. J. Surg. Pathol. 2010; 34; 965-969.

20. Cheng JX, Tretiakova M, Gong C, Mandal S, Krausz T, Taxy JB. Renal medullary carcinoma: rhabdoid features and the absence of INI1 expression as markers of aggressive behavior. Mod. Pathol. 2008; 21; 647-652.

21. Elwood H, Schultz L, Illei PB et al. Immunohistochemical loss of INI-1 expression in collecting duct carcinoma. Mod. Pathol. 2010; 23; 189A.

22. Albadine R, Schultz L, Billis A et al. PAX8(+)/p63(+) immunostaining pattern in renal medullary carcinoma: an intermediate phenotype between urothelial acrcinoma of upper urinary tract and collecting duct carcinoma. Mod. Pathol. 2010; 23; 174A. 